ORIGINAL ARTICLE

\title{
Targets and moving goal posts: changes in waiting times in a UK emergency department
}

\author{
T Locker, S Mason, J Wardrope, S Walters
}

Emerg Med J 2005;22:710-714. doi: 10.1136/emj.2004.019042

See end of article for authors' affiliations a.................

Correspondence to: T Locker; t.locker@ sheffield.ac.uk

Accepted for publication 10 October 2004

\begin{abstract}
Objectives: To describe changes in the case mix, demographics, waiting times (WTs; time from arrival in the emergency department (ED) until seen by a clinician) and treatment times ( $\Pi s$; time from seeing a clinician until leaving the ED) of adult patients presenting to the EDs in Sheffield, UK, between 1993 and 2003.

Design: A retrospective analysis of routinely collected data.

Results: Of a total of 252156 patients included in the study, the proportion of attendees aged 16-29 years decreased from $38.7 \%$ in 1993 to $28.8 \%$ in 2003 (rate of change $(b)=-1.10 \%$ per year, $95 \% \mathrm{Cl}$ $-1.20 \%$ to $-0.82 \%, p<0.001$ ) whereas the proportion aged $80-99$ years increased from $6.2 \%$ to $10.4 \%$ ( $b=0.37 \%$ per year, $95 \% \mathrm{Cl} 0.29 \%$ to $0.45 \%, p<0.001$ ). The proportion of "minors" (patients not arriving by ambulance and subsequently discharged) fell from $71.1 \%$ in 1993 to $60.8 \%$ in 2003 ( $b=-1.04 \%$ per year, $95 \% \mathrm{Cl}-1.36 \%$ to $-0.73 \%, p<0.001)$. WTs increased from a median of 21 minutes in 1993 to 48 minutes in $2003(b=3.5 \mathrm{~min}$ per year, $95 \% \mathrm{Cl} 2.23 \mathrm{~min}$ to $4.77 \mathrm{~min}$, $\mathrm{p}<0.001$ ). The median $\Pi \mathrm{T}$ for minors was unchanged but that for majors (patients arriving by ambulance and admitted to hospital from the ED) increased from 55 to 205 minutes ( $b=11.55$ min per year, $95 \% \mathrm{Cl}$ 6.54 min to 16.55 mins, $p=0.01$ ).

Conclusion: The demographics, case mix, and waiting times of patients presenting to EDs in Sheffield changed considerably over an 11 year period. There is evidence that the service for minor case patients improved slightly at the end of the period studied, but this is possibly at the expense of a deteriorating experience for major case patients.
\end{abstract}

A key target for acute National Health Service (NHS) trusts in the UK at present is improving waiting times (the time from arrival in the emergency department until seen by a clinician) in emergency departments (EDs). The NHS Plan set a target such that by 2004 no one should wait more than four hours from arrival in the ED to admission, discharge, or transfer. ${ }^{2}$ The government envisaged that this would reduce average waiting time in EDs to 75 minutes. Some progress has been made by the sustained investment in new staff and new ways of working. Figures published by the Department of Health show that the proportion of patients spending less than four hours in the ED improved from $77 \%$ in the second quarter of the 2002-03 financial year to $88.1 \%$ in the same period in $2003-04 .^{3}$ Further improvements are going to be challenging and if these are to be achieved, a detailed understanding of the demographics, case mix, and waiting times of patients attending EDs is required. There is growing evidence that the major determinant of ED efficiency is the time that patients have to wait for admission to a hospital bed and that this in turn is determined by the level of bed occupancy. ${ }^{4-8} \mathrm{We}$ have been aware of a deterioration in waiting times in our service over the past 10 years. There are several possible explanations but we hypothesise that a change in case mix, with more patients requiring admission, more elderly patients with complex problems, and delays in moving patients from the ED to inpatient beds, might explain the problem.

This paper aims to describe changes in the case mix, demographics, and waiting times of patients over the age of 16 years presenting to EDs in one city in England over an 11 year period, from 1993 to 2003.

\section{METHOD}

We undertook a retrospective study of routinely collected data from the EDs in Sheffield, a large city in the north of
England. At the start of the study period Sheffield had two EDs, based at the Royal Hallamshire (RHH) and Northern General (NGH) hospitals, which served the adult population and a paediatric ED based at Sheffield Children's Hospital (SCH). These services were reconfigured in 1997 to leave one major adult ED based at the NGH, a nurse led minor injuries unit at the RHH and a paediatric ED at SCH. This reconfiguration has been described in detail elsewhere and has not been shown to have caused any deterioration in ED performance. ${ }^{9}$

For the period 6 April to 5 July for each year from 1993 to 2003 we retrieved information regarding all ED episodes from the ED database. We chose this time period to avoid the times when junior medical staff in the ED rotate into new posts and thus have hopefully avoided any variation resulting from the induction of new staff into the department. We included all new ED episodes either if the patient was aged over 16 years at the time of presentation or if no age was recorded and the patient presented to the ED at the $\mathrm{RHH}$ or NGH. We considered as new episodes all episodes that were neither planned nor unplanned follow up episodes from a previous attendance at the EDs under investigation. Data retrieved included the ED booking-in time, time seen by a decision making clinician (DMC) and time leaving the ED, as well information regarding date of birth, method of arrival, presenting complaint, and disposal.

The DMC could be either a doctor or an emergency nurse practitioner. The ED booking-in time is recorded automatically when a patient registers at the ED. The time seen by a clinician and the time leaving the ED are recorded in the patient's ED notes and these times are then routinely entered into the ED database once the episode is concluded.

Abbreviations: DMC, decision making clinician; ED, emergency department; $T$, treatment time; $W T$, waiting time 
For each episode we calculated the patient's age at presentation. We defined the waiting time (WT) as the time taken from arrival in the ED until the patient was seen by a DMC. Where the WT exceeded 12 hours a null value was recorded as WTs of this length had never occurred in the ED and were therefore most likely to represent errors in the recording of such information at the time of the episode. Similarly, we defined treatment time (TT) as the time from seeing a DMC until the patient left the ED. Null values where recorded where the TT exceeded 24 hours. To identify differences in the WT and TT for patients with different urgencies of presenting complaint we divided the patients into two groups. The "minors" group consisted of those patients who did not arrive by emergency ambulance and were subsequently discharged from the ED. Conversely, the "majors" group consisted of those patients who arrived by emergency ambulance and were admitted to hospital from the ED. We chose these definitions because we thought they would provide the greatest consistency year on year.

For each year we calculated the percentage of patients waiting less than one hour to see a DMC and the percentage of admitted patients spending less than four hours in the ED to allow comparison with figures published by the Audit Commission. ${ }^{1}$

In keeping with local policy, we did not consider it necessary to obtain ethical committee approval for this study as it involved only routinely collected data.

\section{Data analysis}

We analysed the data with Access 2000 (Microsoft Corporation, Redmond WA) and SPSS for Windows 11.5 (SPSS Inc, Chicago, IL). Median and ninetieth percentile values were calculated for the WT and TT for each year. We used linear regression to analyse differences in the rate of increase of ED attendances in Sheffield compared with changes occurring throughout England. We analysed changes in demographics and case mix over time using linear regression to determine their significance. Changes in the median WT and TT were also examined using linear regression to determine if these were related to year of presentation or clinical group (major/minor). For subjects with complete and incomplete WT and TT data the difference in mean age between these groups was assessed using Student's $t$ test. We assessed the differences between these groups regarding the proportion of men, proportion of major cases, and proportion presenting as a result of trauma with the $\chi^{2}$ test.

We excluded episodes with missing variables from each linear regression model where the missing data referred to the variable being tested. Patients were not completely removed from the analysis due to missing data and no attempt was made to extrapolate values for missing variables.

To investigate the trends in median waiting times over the 11 year study period and to correct for differences in age, sex, and the proportions presenting with trauma in each year we used a two stage linear regression procedure. In the first stage, the individual patient's age, sex, and whether or not they were presenting with trauma, were regressed on the patient's original WT (or TT) and then the median unstandardised residuals for each year were calculated. The second stage then used this median residual as the dependent variable, and linear regression was again used to assess if WT (or TT) was related to year of attendance. This two stage procedure had the effect of adjusting the median waiting for any differences in age, sex, and type of trauma over the study period, and still allowed us to use the more appropriate median WT (rather than the mean) as our outcome measure.
The regression coefficient " $b$ " represents the rate of change in the parameter estimate (for example median waiting time) over the 11 year time period.

\section{RESULTS}

During the period studied there were 252156 new patient episodes. The average increase in total attendances in Sheffield during the period studied was $1.3 \% \quad(n=969$ attendances) per annum (95\% confidence interval (CI) $-4.2 \%$ to $6.8 \%$ ). This is smaller than the average annual increase nationally of $1.7 \%(\mathrm{n}=0.2$ million attendances $)$ per annum. ${ }^{10}$ Considering the annual percentage change in Sheffield and that in England together, linear regression demonstrates that the percentage change each year was related neither to year of attendance $(b=-0.6 \%, 95 \%$ CI $-0.91 \%$ to $0.79 \%, \mathrm{p}=0.88$ ) nor to site (Sheffield or England) $(b=0.86 \%, 95 \%$ CI $-4.03 \%$ to $5.74 \%, p=0.72)$. This showed that is there had been no significant alteration in the percentage change in attendances each year and no difference between the rate of increase in Sheffield and England.

\section{Patient age}

The age profile changed, with the proportion of attendees aged $16-29$ years falling from $38.7 \%(n=9089)$ in 1993 to $28.8 \%(\mathrm{n}=7775)$ in $2003(b=-1.10 \%, 95 \% \mathrm{CI}-1.20 \%$ to $-0.82 \%, \mathrm{p}<0.001)$. There was a corresponding increase in the proportion of patients in older age groups, most notably in those aged 80-99 years of age; this age group accounting for $6.2 \%(\mathrm{n}=1437)$ of attendances in 1993 and $10.4 \%(\mathrm{n}=2695)$ in 2003 ( $b=0.37 \%, 95 \%$ CI $0.29 \%$ to $0.45 \%, \mathrm{p}<0.001)$.

\section{Presenting complaint}

The proportion of new patients arriving by emergency ambulance increased from $22.7 \%(\mathrm{n}=5080)$ in 1993 to a peak of $31.6 \%(\mathrm{n}=7838)$ in $2002(b=0.93 \%, 95 \%$ CI $0.62 \%$ to $1.24 \%, \mathrm{p}<0.001)$. The reasons patients attend an ED can be broadly divided into problems arising due to trauma and those due to other causes. Trauma was responsible for the majority of attendances in the study, accounting for a maximum of $59.4 \%(\mathrm{n}=11599)$ of episodes in 1997 and falling to $52.9 \%(\mathrm{n}=13144)$ in $2003(b=-0.46 \%$, 95\% CI $-8.7 \%$ to $-0.04 \%, \mathrm{p}=0.04)$. This pattern was apparent in all age groups but was most marked among older patients.

The proportion of cases defined as "minor" fell from $71.1 \%$ $(\mathrm{n}=15945)$ in 1993 to $60.8 \%(\mathrm{n}=15096)$ in 2003 $(b=-1.04 \%, 95 \%$ CI $-1.36 \%$ to $-0.73 \%, \quad p<0.001)$. Correspondingly "major" cases accounted for $9.2 \%$ $(\mathrm{n}=2052)$ of episodes in 1993 increasing to $14.0 \%$ $(\mathrm{n}=3482)$ in $2003(b=0.53 \%, 95 \%$ CI $0.30 \%$ to $0.76 \%$, $\mathrm{p}=0.01)$.

\section{Missing waiting time and treatment time data}

Patients with complete data for WT and TT accounted for $84.8 \%(n=213911)$ of the sample studied. The mean age of patients with complete data was 44.4 years compared to 41.5 years for patients with incomplete data (WT or TT missing) and this difference was statistically significant $(t=25.09$,

Table 1 Comparison of percentage of men, major cases, and cases due to trauma for patients with complete and incomplete WT and TT data

\begin{tabular}{|c|c|c|c|c|}
\hline & Complete & Incomplete & $\chi^{2}$ & $\mathbf{p}$ \\
\hline Men & 55.3 & 58.0 & 103.1 & $<0.001$ \\
\hline Major cases & 13.3 & 8.6 & 649.0 & $<0.001$ \\
\hline Cases due to trauma & 59.6 & 39.8 & 5181.2 & $<0.001$ \\
\hline
\end{tabular}




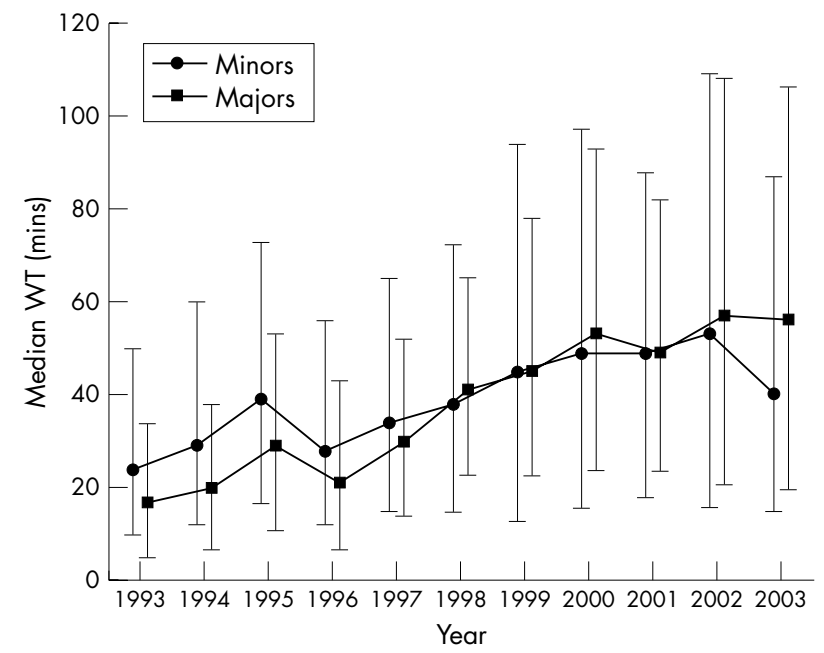

Figure 1 Changes in median waiting time (WT). Vertical bars represent the interquartile range of WTs. Minors: patients who did not arrive by emergency ambulance and were subsequently discharged from the ED; majors: patients who arrived by emergency ambulance and were admitted to hospital from the ED.

$\mathrm{df}=53911.8, \mathrm{p}<0.001)$. The differences between these groups with regard to the proportion of men, cases resulting from trauma, and proportion of major cases are shown in table 1.

\section{Waiting time}

Waiting times altered considerably, with a median of 21 minutes and ninetieth percentile WT of 83 minutes in 1993, rising to 48 and 165 minutes, respectively in 2003 $(b=3.5 \mathrm{~min}, 95 \%$ CI $2.23 \mathrm{~min}$ to $4.77 \mathrm{~min}, \mathrm{p}<0.001)$. Figure 1 demonstrates change in the median WT for minor and major cases. In 1993 the median WT for a minor was 24 minutes and for a major was 19 minutes. In 2003 this pattern was reversed, with the median WT increasing to 40 and 58 minutes, respectively, for minor and major cases. Correcting for changes in age, sex, and the proportion of patients presenting as a result of trauma, linear regression demonstrates that changes in median WT are related to year of attendance $(b=3.41 \mathrm{~min}, 95 \%$ CI 2.60 to 4.23 , $\mathrm{p}=<0.001)$ but not to clinical group-that is, major or minor $(b=0.43 \mathrm{~min}, 95 \% \mathrm{CI}-10.89$ to $11.74, \mathrm{p}=0.94)$.

\section{Treatment time}

The distribution of TT also changed markedly. The median TT in 1993 was 30 minutes with a ninetieth percentile TT of 90 minutes. In 2003 these parameters had both increased with a median TT of 45 minutes but most notably a ninetieth percentile TT of 242 minutes ( $b=0.96 \mathrm{~min}, 95 \%$ CI $0.33 \mathrm{~min}$ to $1.60 \mathrm{~min}, \mathrm{p}=0.007)$. The median TT for major and minor cases differed. Figure 2 demonstrates that the median TT for minors changed little from 1993 to 2003, varying between 25 and 34 minutes. In contrast the median TT for majors almost quadrupled from 55 minutes in 1993 to 205 minutes in 2003.

Correcting for changes in age, sex, and the proportion of patients presenting as a result of trauma, linear regression shows that for minor cases median TT was not related to year of attendance ( $b=0.13 \mathrm{~min}, 95 \% \mathrm{CI}-0.38 \mathrm{~min}$ to $0.65 \mathrm{~min}$, $\mathrm{p}=0.58$ ) but a relation between median TT and year did exist for major cases $(b=11.46 \mathrm{~min}, 95 \%$ CI $6.68 \mathrm{~min}$ to $16.24 \min , \mathrm{p}<0.001)$.

\section{Outcome}

The disposition of patients from the ED is shown in table 2 . The number of patients admitted to hospital during the three

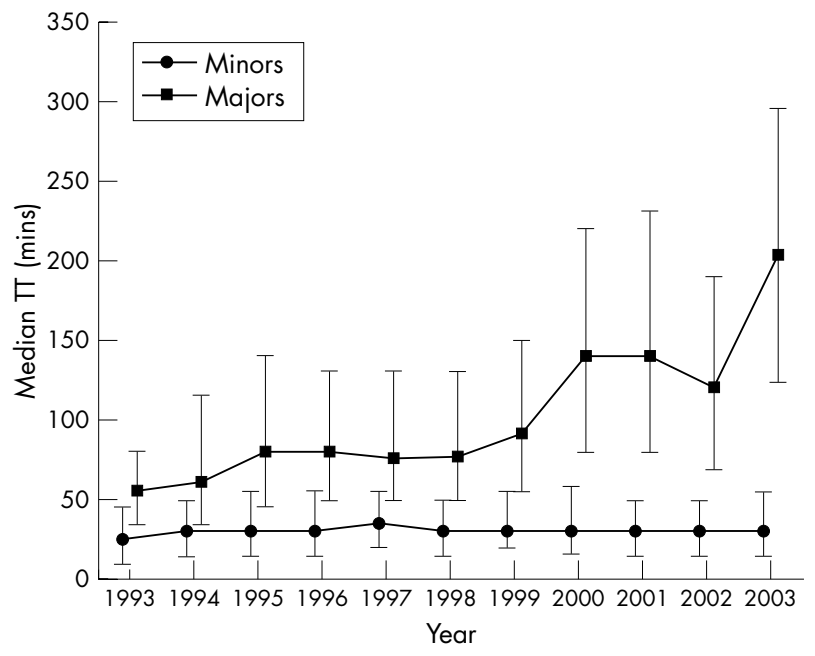

Figure 2 Changes in median treatment time (TT). Vertical bars represent the interquartile range of $\Pi \mathrm{s}$. Minors: patients who did not arrive by emergency ambulance and were subsequently discharged from the ED; majors: patients who arrived by emergency ambulance and were admitted to hospital from the ED.

Table 2 Disposal from the ED. Per cent of total new attendances in parentheses

\begin{tabular}{rrrllll}
\hline Year & Not known & Discharged & \multicolumn{1}{l}{ Admitted } & \multicolumn{1}{l}{ Died } & \multicolumn{1}{l}{ Total } \\
\hline 1993 & $105(0.5)$ & $18795(83.8)$ & $3358(15.0)$ & $160(0.7)$ & 22418 \\
1994 & $199(0.8)$ & $19036(81.1)$ & $4106(17.5)$ & $142(0.6)$ & 23483 \\
1995 & $143(0.6)$ & $19150(80.9)$ & $4277(18.1)$ & $113(0.5)$ & 23683 \\
1996 & $477(2.1)$ & $18711(81.5)$ & $3666(16.0)$ & $111(0.5)$ & 22965 \\
1997 & $537(2.8)$ & $14923(76.4)$ & $4006(20.5)$ & $58(0.3)$ & 19524 \\
1998 & $211(0.9)$ & $17687(79.0)$ & $4435(19.8)$ & $61(0.3)$ & 22394 \\
1999 & $25(0.1)$ & $17319(79.6)$ & $4363(20.1)$ & $53(0.2)$ & 21760 \\
2000 & $191(0.8)$ & $17395(77.4)$ & $4845(21.6)$ & $42(0.2)$ & 22473 \\
2001 & $246(1.0)$ & $18555(77.9)$ & $4976(20.9)$ & $52(0.2)$ & 23829 \\
2002 & $254(1.0)$ & $19356(78.1)$ & $5109(20.6)$ & $68(0.3)$ & 24787 \\
2003 & $267(1.1)$ & $18872(76.0)$ & $5629(22.7)$ & $72(0.3)$ & 24840 \\
\hline
\end{tabular}

Table 3 Changes in ED staffing. (Staffing figures shown are for numbers of whole time equivalents unless stated otherwise)

\begin{tabular}{lcc}
\hline & 1993 & 2003 \\
\hline Consultants & 4 & 4.3 \\
Registrars & 3 & 4 \\
Senior house officers & 19 & 18 \\
Pre-registration house officers & 0 & 1 \\
General practitioner clinical assistants (sessions) & 13 & 12 \\
Trust grade/clinical fellows & 0 & 4 \\
Emergency nurse practitioners & 0 & 9.64 \\
\hline
\end{tabular}

month period studied for each year increased from 3358 $(15.0 \%)$ in 1993 to $5629(22.7 \%)$ in $2003(b=0.64 \%, 95 \%$ CI $0.38 \%$ to $0.89 \%, \mathrm{p}<0.001)$.

\section{Resources available}

The numbers of medical staff and emergency nurse practitioners working in the EDs in Sheffield in 1993 and 2003 are shown in table 3. The number of acute hospital beds available in Sheffield fell from 2900 in 1993 to 2074 in 2003. 


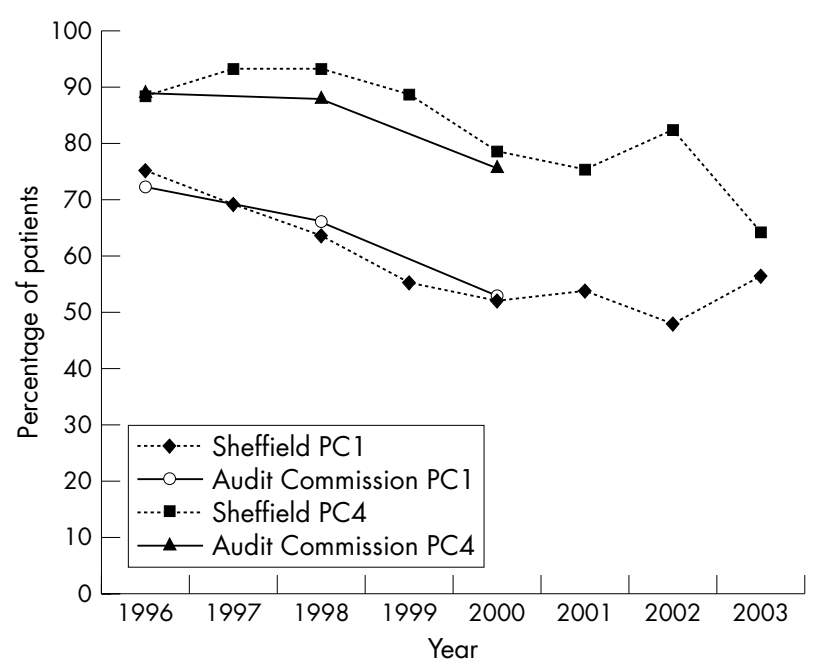

Figure 3 Changes in the percentage of patients waiting less than one hour to be seen (PC1) and the percentage of admitted patients admitted within four hours (PC4) for patients included in this study (Sheffield PCl and PC4) compared to the findings of the Audit Commission (Audit commission $\mathrm{PCl}$ And $\mathrm{PC}$ ). $\mathrm{PCl}$ : patient seen by a DMC within one hour of arrival; PC4: admitted patient admitted within four hours of arrival.

\section{Comparison with the findings of the Audit Commission} Our study showed that the percentage of patients seeing a DMC within one hour of arrival (PCl) fell from 75.2\% in 1996 to $56.4 \%$ in 2003 . The percentage of admitted patients admitted within four hours of arrival (PC4) declined from $88.2 \%$ to $64.1 \%$ over the same time period. Figure 3 shows how these changes compare to changes in the median PCl and PC4 found by the Audit Commission in their reviews of EDs in England and Wales. ${ }^{1}$

\section{DISCUSSION}

The above findings demonstrate that there were significant changes in the demographics and case mix of patients attending the EDs in Sheffield from 1993 to 2003. Marked changes also occurred in both the WTs and TTs. WTs increased for both minor and major patients but only recently has the median WT for a minor case been less than that for a major. In the UK, a number of strategies have been implemented nationally to improve WT for minors, including the establishment of separate minor injury units and "streaming" of minor cases. ${ }^{11}{ }^{12}$ A separate minor injury unit has been present in Sheffield since 1997 and in 2003 a separate stream for minor injuries has been in operation part time in the ED of the NGH. It would appear that these services are beginning to have a significant impact upon the service for 'minors' in Sheffield. Although it may appear unethical and lacking in good clinical sense that minors are being seen quicker than major cases, the improvement is probably due mainly to the use of new money to employ minor injury emergency nurse practitioners. The result is that it appears that WTs, at least for minors, are beginning to fall.

The median TT for minor injuries remained static over the period studied whereas that for majors increased considerably. As far as we are aware this finding has not been previously demonstrated. The service received by minor cases will be predominantly under the control of the ED, with little recourse to other specialties or specialised diagnostic services in most cases. This may not be the case for major cases. It was not possible in this study to determine what events occurred during the TT. Further work would obviously be vital to determine why the TT of major patients has increased so greatly. This time period will include the time taken to assess the patient by the ED clinician and obtain any necessary investigations, as well as the time from referral to transfer to an inpatient bed. Whatever the cause, the service received by major cases continues to deteriorate at an alarming rate.

The numbers of patients attending the ED is increasing, but the proportion of the patients subsequently discharged has fallen considerably. If the figures presented above are extrapolated to an entire year's ED attendances in Sheffield, an extra 9000 adult patients per annum are being admitted to hospital from the ED, whereas the number of acute hospital beds fell during the study period from 2900 to 2074. Possibly of greater importance is the fact that the majority of these patients are now being admitted to one hospital rather than ED admissions being distributed across two hospitals, as was the case prior to the reorganisation of services in 1997. These changes may account in part for the increase in TT for major patients if inpatient beds are not available to receive such patients from the ED.

It is difficult to interpret how the changes in staffing have influenced the findings presented here. Although numbers of staff have increased, particularly provision of emergency nurse practitioners, the service provided has altered considerably.

Our aim in the present study was to describe the changes that have occurred. To make inferences about causation is beyond the scope of this paper and would not be possible with the data collected.

The study has some weaknesses. The data were retrospective but data collection has been one of the major objectives of the Sheffield emergency service and steps were taken to ensure common data collection practices across all sites, such as the training of medical and nursing staff in the recording of data and training of administrative staff in data entry. Sheffield has also had a major change in its services during this period. However this change was subject to a rigorous prospective audit of waiting and admission times. ${ }^{9}$ We did not detect any deterioration in these measures, indeed there was a slight improvement. The Audit Commission has examined WTs in other EDs in the UK and found a similar deterioration in the majority of departments. ${ }^{1}$ It has also demonstrated a decrease in the proportion of patients admitted within four hours of arrival over time. The Audit Commission's findings are based on data collected since 1996. Our findings, presented above, would suggest the changes in Sheffield are not unique or isolated. The deterioration in WTs in Sheffield would appear to mirror changes throughout England and Wales. It also appears that many EDs in other countries are experiencing similar problems. ${ }^{13} 14$

\section{CONCLUSION}

We have confirmed that WTs and TTs in Sheffield have deteriorated over the past 10 years. Recent innovation and investment is showing improvement for "minor cases". The TTs for major cases have increased markedly probably due to an increase in the volume of elderly patients and numbers of patients requiring admission. We postulate that a change in the way illness is dealt with in the community is resulting in more patients being brought to hospital and admitted. If the UK government wishes to hit the targets for emergency care then it must concentrate on emergency primary care. However, the recent changes to the General Medical Services contract and the withdrawal of many general practitioners from emergency primary care make it difficult to envisage how this will be achieved.

\section{CONTRIBUTORS}

TL, JW, and SM conceived the study. TL and SW performed the analysis. All authors contributed to the writing and editing of the manuscript. TL is the guarantor. 


\section{Authors' affiliations}

T Locker, S Mason, Medical Care Research unit, School of Health and Related Research, University of Sheffield, Regent Court, 30 Regent Street, Sheffield, S1 4DA, UK

J Wardrope, Emergency Department, Northern General Hospital, Herries Road, Sheffield, S5 7AU, UK

S Walters, Sheffield Health Economics Group, School of Health and Related Research, University of Sheffield, Regent Court, 30 Regent Street, Sheffield, S1 4DA, UK

This study was conducted as part of a study funded by the National Coordinating Centre for NHS Service Delivery and Organisation Research and Development (SDO). The views expressed are those of the authors and not necessarily those of the funding body.

Competing interests: At the time of submission/acceptance of this paper $J$ Wardrope was an editor of this journal.

\section{REFERENCES}

1 Audit Commission. Accident and Emergency. Review of National Findings. London: Audit Commission, October, 2001.

2 Department of Health. The NHS Plan. London: DoH, 2000.

3 Total time spent in A\&E from arrival to admission, transfer or discharge, NHS organisations in England, 2003-04 July to Sept (Q2). Department of Health dataset QMAE. December, 2003. London: DoH.
4 Proudlove NC, Gordon K, Boaden R. Can good bed management solve the overcrowding in accident and emergency departments. Emerg Med J 2003;20:149-55

5 Derlet RW, Richards JR, Kravitz RL. Frequent overcrowding in US emergency departments. Acad Emerg Med 2001;8:151-5.

6 Bazarian JJ, Schneider SM, Newman VJ, et al. Do admitted patients held in the emergency department impact the throughput of treat-and-release patients? Acad Emerg Med 1996;3:1113-18.

7 Forster AJ, Stiell I, Wells G, et al. The effect of hospital occupancy on emergency department length of stay and patient disposition. Acad Emerg Med 2003;10:127-33.

8 Dunn R. Reduced access block causes shorter emergency department waiting times: an historical control observational study. Emerg Med J 2003;15:232-8.

9 Simpson AN, Wardrope J, Burke D. The Sheffield experiment: the effects of centralising accident and emergency services in a large urban setting. Emerg Med J 2001; 18:193-7.

10 Attendances at A\&E departments, England, 1987-88 to 2001-02, Department of Health form KH09. London: DoH.

11 Cooke MW, Wilson S, Pearson S. The effect of a separate stream for minor injuries on accident and emergency department waiting times. Emerg Med $J$ 2002;19:28-30.

12 NHS Modernisation Agency. Improvements in Emergency Care: Case Studies. London: NHS Modernisation Agency, October, 2002.

13 Schafermeyer RW, Asplin BR. Hospital and emergency department crowding in the United States. Emerg Med (Freemantle), 2003;15:22-7.

14 Schull MJ, Szalai JP, Schwartz B, et al. Emergency department overcrowding following systematic hospital restructuring: trends at twenty hospitals over ten years. Acad Emerg Med $2001 ; 8: 1037-43$. 